\title{
Impact of oral and intramuscular administration amoxicillin on the selection of amoxicillin-resistant Enterobacteriaceae in the digestive flora of piglets
}

\author{
Kouadio I.K1,2, Guessennd N.K.2,3, Dadié A. ${ }^{1}$, Koffi K.E.4, Ouattara M.B ${ }^{1,2}$, Tiékoura B.K. ${ }^{2}$, Konan \\ F.K. ${ }^{2}$, Gbonon C.V. ${ }^{2}$ Djé M.K. ${ }^{1}$, Dosso M.2,3. \\ 1. Laboratory of Biotechnology and Microbiology of Food, Department of Technology and Foods Sciences, Nanguy \\ Abrogoua University, Abidjan, 02 BP 801 Abidjan 02, Côte d'Ivoire \\ 2. National Reference Center for Antibiotics, Department of Bacteriology and Virology, Pasteur Institute, Abidjan, 01 \\ BP 490 Abidjan 01, Côte d'Ivoire \\ 3. Laboratory of Bacteriology and Virology of Faculty of Medical Science, Felix Houphouët-Boigny University, Abidjan, \\ 01 BP V34 Abidjan 01, Côte d'Ivoire \\ 4. Unity of Animal Resources Management, Environment and Health Department, Pasteur Institute, Abidjan, 01 BP \\ 490 Abidjan 01, Côte d'Ivoire \\ E-mail address of corresponding author: kouaminos@yahoo.fr
}

Original submitted in on 29th May 2017. Published online at www.m.elewa.org on $30^{\text {th }}$ June 2017 https://dx.doi.org/10.4314/jab.v114i1.13

\begin{abstract}
Objective: The aim of this study was to evaluate the level of selection of amoxicillin-resistant Enterobacteriaceae in the digestive microbiota of piglets during oral and intramuscular administration of amoxicillin.

Methodology and Results: Enumeration of Enterobacteriaceae was carried out on MacConkey agar with and without amoxicillin. $E$. coli isolates were identified and subjected to antimicrobial susceptibility test. The percentages of amoxicillin-resistant Enterobacteriaceae before treatment were between 10-13\% for the three groups of piglets. After starting treatment of amoxicillin, from day 1 to day 4 , the percentages of resistant Enterobacteriaceae were between 54 to $81 \%$ for the intramuscular treated groups and 58 to $87 \%$ for those treated orally. In the control group, percentage was $11 \%$. During days of treatments, the percentage of amoxicillin-resistant $E$. coli strains to the associated antibiotics evolved during the treatments. In the control piglets, the percentages of $E$. coli resistant antibiotics did not increase.

Conclusion and application of findings: Administration of oral amoxicillin resulted in a greater selection of resistant enterobacteria in the digestive microbiota of piglets than those induced by the intramuscular route. Escherichia coli, an important strain in animal and human pathology, was the target of detection in this selection of these resistant enterobacteria. They constitute a public health risk due to the transfer of resistance genes from pathogenic bacteria of porcine origin to the human digestive flora via the food chain. The results obtained from this study are part of a bacterial resistance monitoring policy that will prevent pig farmers from Côte d'Ivoire, the majority user of these antibiotics.
\end{abstract}

Keywords: Piglets, Enterobacteriaceae, Amoxicillin, Resistance, Digestive microbiota 


\section{INTRODUCTION}

The use of antibiotics in the piggery is about $60 \%$ of the world consumption of antibiotics in the animal sector (FEDESA, 2000). The massive uses of these antimicrobials contribute an important selection of resistant or multi-resistant bacteria in the digestive microbiota of pigs (Sunde et al., 1998). A transfer of resistance genes from commensal or pathogenic bacteria of animal origin to the human digestive flora via the food chain or the environment is now a real public health problem (Phillips and Casewell, 2004; Sanders et al., 2011). The human intestinal flora would thus be enriched with genetic determinants of resistance of animal origin, potentially transmissible to pathogenic bacteria (Salyers et al., 2004). Bacteria isolated in animals and humans share the same mechanisms of resistance. This is an argument in favour of the lack of sealing between bacterial populations of human and animal origin. Beta-lactams are widely used in veterinary medicine and beta-lactamases identified in Enterobacter

\section{MATERIAL AND METHODS}

Experimental study and collection of samples: Six (6) Large White weaned piglets, 4-week-old that had never received antibiotics were used for the experiments. The piglets were weighed using a scale (BSCl RoHS, Nops $®$, China) to determine their weights. These piglets were housed separately in individual pens all through the experiments. Amoxicillin was administered at a dose of 1 $\mathrm{ml} / 10 \mathrm{~kg}$ of body weight for 5 days by using two routes: oral route and intramuscular route. Three groups of piglets were formed: a first group of 2 piglets $(n=2)$ received amoxicillin (Vetrimoxin ${ }^{\circledR}$ ) by intramuscular route, a second group of 2 piglets $(n=2)$ received amoxicillin (Cofamox ${ }^{\circledR} 20$ ) by oral route and a third group of 2 piglets $(\mathrm{n}=2)$ did not receive antibiotics during the experimental study, which served as controls. Fecal samples were collected from each piglet before treatment (day 0 ) and after 1, 2, 3 and 4 days treatments. A total of 30 fecal samples were collected during the study. The samples were taken from a sterile coproculture box and transported at $4{ }^{\circ} \mathrm{C}$ in the laboratory for microbiological analysis.

Phenotypic evaluation of the level of resistance to amoxicillin: $5 \mathrm{~g}$ of faeces from each piglet was taken and emulsified in $45 \mathrm{ml}$ peptone water including $30 \%$ glycerol. After homogenization, tenfold serial dilution of the filtrate was prepared, $100 \mu$ l of diluted sample of dilution was iaceae of animal origin are identical to those identified in Enterobacteriaceae of human origin ( $\mathrm{Li}$ et al., 2007). Beta-lactam as amoxicillin is therefore a molecule of choice for the treatment of bacterial respiratory diseases in pigs (Pijpers et al., 1989). However, beta-lactams in general and aminopenicillin in particular are the most widely used therapeutic class in human medicine (Livermore, 1995). Given the probable public health risks associated with the consumption of porcine foodstuffs with the transfer of resistance genes to the human digestive flora, it was considered appropriate to consider an assessment of the level of selection of Beta-lactam-resistant Enterobacteriaceae in the digestive flora of piglets during oral and intramuscular administration of this antibiotic. The strains of Escherichia coli were the target enterobacterium and are often used for its importance in animal and human pathology (Sorum and Sunde, 2001).

spread on MacConkey agar (Bio-Rad, Marne la Coquette, France) containing 0 and $16 \mu \mathrm{g} / \mathrm{ml}$ of amoxicillin. The plates were incubated at $37^{\circ} \mathrm{C}$ for 24 hours. Presumptive colonies of enterobacteria on agar containing $16 \mu \mathrm{g} / \mathrm{mL}$ of amoxicillin were classified as resistant according to the standard value of the Committee of Veterinarians of the French Society of Microbiology (CASFM, 2014). Enterobacteriaceae counts from both plates were used to determine the percentage of amoxicillin-resistant Enterobacteriaceae at each sampling time. For each sample, five presumptive colonies of $E$. coliwere selected from Petri dishes containing the MacConkey agar with amoxicillin. A subculture was carried out on Rapid $E$. coli 2 medium (Bio-Rad, Marne la Coquette, France) in order to obtain pure cultures. The plates were incubated at $37^{\circ}$ $C$ for 24 hours. The pure presumptive colonies of $E$. coli were identified by using biochemical test of Leminor. The percentage (R) of amoxicillin-resistant Enterobacteriaceae was determined according to the following equation:

\section{$E_{R}$}

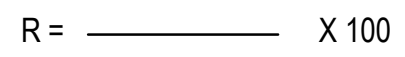

$\mathrm{E}_{\mathrm{T}}$ 
$E_{R}$ : Number of colonies of Enterobacteriaceae on MacConkey agar with amoxicillin

$\mathrm{E}_{\mathrm{T}}$ : Number of colonies of Enterobacteriaceae on MacConkey agar without amoxicillin

Antibiotics susceptibility test: Antibiotics susceptibility test of $E$. coli strains were performed by using the disk diffusion method on agar medium according to the CASFM standard. Fourteen (14) antibiotic disks were used for the study: amoxicillin $(25 \mu \mathrm{g})$, amoxicillin + clavulanic acid $(20 / 10 \mu \mathrm{g})$, cefotaxime $(30 \mu \mathrm{g})$, ceftriaxone $(30 \mu \mathrm{g})$, cefoxitin $(30 \mu \mathrm{g})$, ciprofloxacin $(5 \mu \mathrm{g})$, nalidixic acid $(30 \mu \mathrm{g})$, tetracycline $(30 \mu \mathrm{g})$, trimethoprim / sulfamethoxazole (1.25 / $23.75 \mu \mathrm{g})$, chloramphenicol (30 $\mu \mathrm{g})$, kanamycin $(30 \mu \mathrm{g})$, streptomycin $(10 \mu \mathrm{g})$, colistin $(50$ $\mu \mathrm{g})$. The antibiogram was performed on Mueller Hinton agar (Bio-Rad, Marne la Coquette, France). Interpretation of the results was done according to the rules and recommendations of the veterinarians of the Antibiogram Committee of the French Society of Microbiology (CASFM, 2014). E. coli ATCC 25922 was used as the control strain. The percentage $(\mathrm{Pc})$ of $E$. coli-resistant antibiotics was determined according to the following equation:

\section{RESULTS}

Percentages of amoxicillin-resistant Enterobacter iaceae: Figure 1 shows the percentage of amoxicillinresistant Enterobacteriaceae for each mode of amoxicillin administration. Before the start of treatment (day D0), the percentage of amoxicillin-resistant Enterobacteriaceae was estimated at $10-13 \%$ for all groups of piglets. From day D1 to D4, the percentages of Enterobacteriaceae exceeded the $50 \%$ threshold ranging from $54 \%$ to $81 \%$ for intramuscularly treated groups and from $58 \%$ to $87 \%$ for groups treated orally. The percentages of resistant

$$
E_{\text {ATB }}
$$

$\mathrm{Pc}=$ $\mathrm{X} 100$

$E_{T T}$

$E_{\text {ATB: }}$ Number of strain identified E. coli resistant to an associated antibiotic from presumptive colonies of Enterobacteriaceae selected on MacConkey medium with amoxicillin

$\mathrm{E}_{\mathrm{TT}}$ : The five presumptive colonies of Enterobacteriaceae selected on the MacConkey medium with amoxicillin

Statistical analysis: Statistical analysis of the data was performed using GraphPad Prism® 5.01 software (Microsoft ${ }^{\circ}$, USA). Comparisons of the various percentages of amoxicillin-resistant Enterobacteriaceae in the treated and control groups were performed using the Tukey's multiple comparison assay. The difference between the constituted groups was considered significant at $p<0.05$.

enterobacteria of the controls did not exceed $11 \%$. The percentages of amoxicillin-resistant Enterobacteriaceae determined during oral administration of this antibiotic were greater than those of intramuscular treatments. Statistical analysis was indicated a significant increase $(p$ $<0.05$ ) in the percentage of amoxicillin-resistant enterobacteria in the oral and intramuscular groups with the control group, but no significant difference between the group Treated orally and the group treated intramuscularly. 


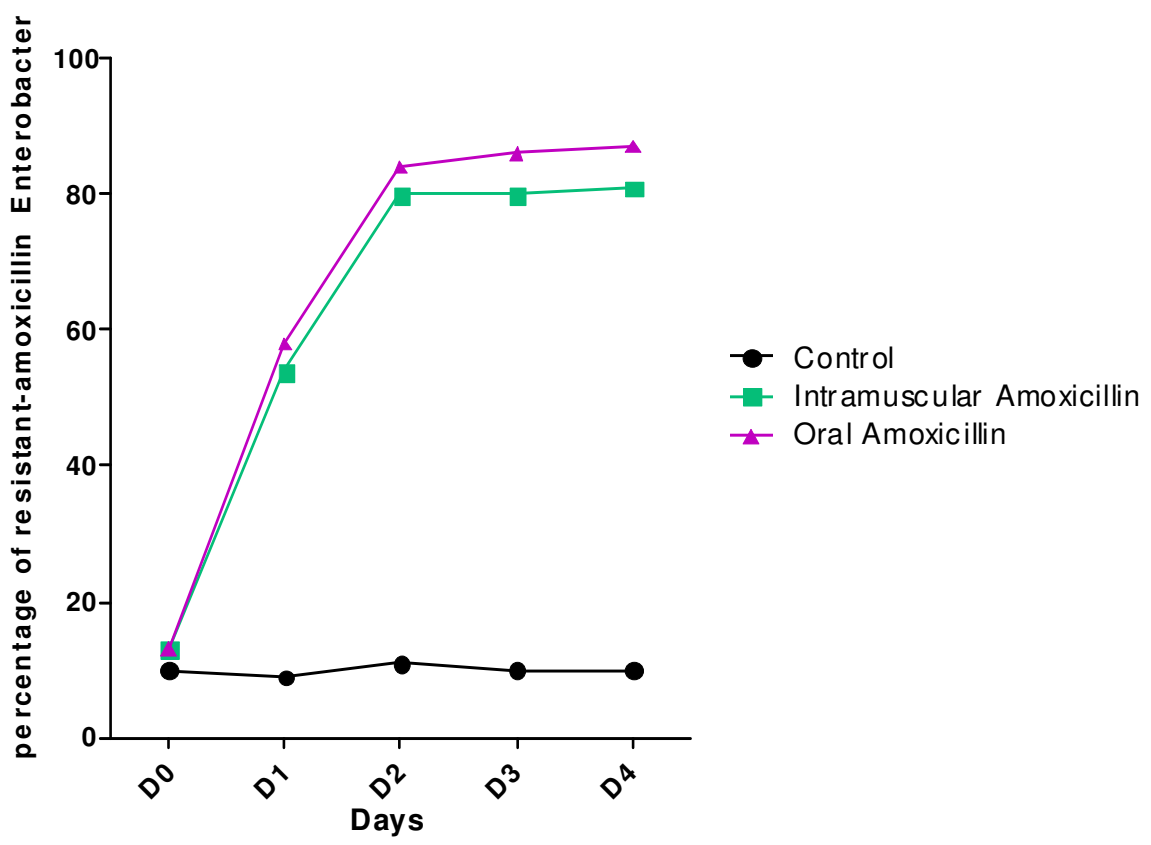

Figure 1: Percentage of amoxicillin-resistant Enterobacteriaceae for each mode of amoxicillin administration

Associated amoxicillin resistance: Table 1 shows the percentage of $E$. coli isolates resistant to amoxicillin, ceftriaxone, nalidixic acid, tetracycline, chloramphenicol, trimethprime sulfamethoxazole, streptomycin, gentamicin and colistin. None of $E$. coli isolates was resistant to colistin. Before amoxicillin treatment (day D0) in all constituted groups, the percentages of $E$. coli resistant to tetracycline was $60 \%$ and $45 \%$ to trimethoprim sulfamethoxazole were the most important percentage. The percentage of $25 \%$ of $E$. coli resistant to streptomycin was the third most important before treatment. As for the percentages of strains resistant to amoxicillin, nalidixic acid, chloramphenicol and gentamicin, they did not exceed $15 \%$. From the first to the fourth day of treatment, the percentages of strains resistant to amoxicillin, trimethoprim sulfamethoxazole, streptomycin and tetracycline have evolved to over $50 \%$ in antibiotic-treated groups. The percentage of strains resistant to amoxicillin and tetracycline exceeded $90 \%$ from the third day of treatment. The percentages of strains resistant to nalidixic acid, chloramphenicol and gentamicin also slightly changed and did not exceed 25\% resistance.

Table 1: Percentage of amoxicillin-resistant E. coli strains in control and treated piglets

Percentage of amoxicillin-resistant E. coli strains in control and treated piglets

\begin{tabular}{l|c|c|c|c|c|c|c|c|c|c}
\hline \multicolumn{1}{c}{ Before treatment } & \multicolumn{9}{c}{ After treatment } & \multicolumn{2}{c}{ Day 3 } & \multicolumn{2}{c}{ Day 4 } \\
\hline & \multicolumn{2}{c}{ Day 0 } & \multicolumn{2}{c}{ Day 1 } & \multicolumn{2}{c|}{ Day 2 } & \multicolumn{2}{c}{ Dat } \\
\hline ATB & for treated & control & treated & control & treated & control & treated & control & treated & control \\
\hline AMX & 15 & 11 & 50 & 2 & 80 & 3 & 95 & 7 & 98 & 3 \\
\hline CRO & 0 & 0 & 3 & 0 & 0 & 0 & 2 & 0 & 5 & 0 \\
\hline NAL & 10 & 15 & 10 & 16 & 10 & 17 & 10 & 16 & 18 & 16 \\
\hline TET & 60 & 67 & 90 & 60 & 93 & 66 & 95 & 61 & 97 & 60 \\
\hline CHL & 1 & 0 & 11 & 0 & 10 & 0 & 12 & 0 & 15 & 40 \\
\hline SXT & 45 & 47 & 56 & 50 & 62 & 50 & 65 & 40 & 75 & 35 \\
\hline SMN & 25 & 22 & 54 & 25 & 60 & 20 & 69 & 20 & 80 & 25 \\
\hline GMI & 10 & 15 & 20 & 15 & 25 & 20 & 20 & 20 & 25 & 15 \\
\hline CST & 0 & 0 & 0 & 0 & 0 & 0 & 0 & 0 & 0 & 0 \\
\hline
\end{tabular}

ATB: Antibiotic, AMX: Amoxicillin, CRO: Ceftriaxone, NAL: Nalidixic acid, TET: Tetracyclin, CHL: Chloramphenicol, SXT: Trimethoprim sulfamethoxazole, SMN: Streptomycin, GMI: Gentamicin, CST: Colistin 


\section{DISCUSSION}

The results determined in this study showed a considerable increase in amoxicillin-resistant Enterobacteriaceae in the digestive tract of piglets during oral and intramuscular administration of antibiotics. Several studies have shown that in pigs, as in other animals, antibiotic administration increases the prevalence of resistance in the bacteria of the commensal digestive flora (Sorum and Sunde, 2001, Belloc et al., 2005; Kempf et al., 2014). When antibiotics are administered, digestive bacteria that are resistant to the antibiotic are selected, and these resistant bacteria can then colonize the digestive tract (Sorum and Sunde, 2001). Sunde et al. (1998) reported the prevalence of resistance in $E$. coli isolated from pigs in 10 farms with a history of antibiotic use for different therapeutic purposes. They showed that the highest percentage of resistant bacteria was found in farms where the use of antibiotics was considered high. The number of selected Enterobacteriaceae resistant to amoxicillin in the digestive flora of piglets following the administration of oral antibiotic in the study was superior to those administered intramuscularly. This result shows that the administration of oral antibiotics in animals obviously leads to an important selection of resistant enterobacteria in their digestive microbiota compared to those induced by intramuscular administration. Other studies conducted by Bibbal et al. (2007) and Zhang and Huang (2013) reported that oral administration of antibiotics resulted in the greatest exposure of resistant digestive bacteria. Massive exposure of bacteria to the digestive tract leading to emergence of resistance strains because of oral antibiotic treatment could be explained because of their direct contact with the antibiotic (Bibbal et al., 2007). In fact, the digestive content can be the origin of various phenomena of fixation, which render the antibiotic inactive

\section{CONCLUSION}

Control of the sanitary quality of livestock requires the control of bacterial infections, which uses antibiotics whose use promotes a selection of resistant bacteria in the digestive microbiota of animals. The administration of these antibiotics takes place in several ways. The administration of oral amoxicillin in these animals therefore leads to a greater selection of resistant enterobacteria in their digestive microbiota than those
(Jansen et al., 1992). The study of associated resistance of amoxicillin in E. coli isolates within the amoxicillinresistant enterobacteria prior to initiation of treatment revealed that few strains were susceptible to all antibiotics tested. The percentage of tetracycline resistant $E$. coli was $60 \%$ and trimethoprim sulfamethoxazole was $45 \%$. These results are consistent with those obtained in the study of Bibbal et al. (2009) in France where, prior to ampicillin treatment of piglets, the frequency of tetracycline-resistant E. coli was $69 \%$ and $46 \%$ for sulphonamide. However, the slight difference observed with ampicillin, which registered a resistance of $6 \%$ before treatment to this antibiotic, compared to a resistance of $15 \%$ to amoxicillin in this study. After the beginning of the treatment, the results showed that in the group of piglets treated with antibiotics, the number of strains of $E$. coli resistant to the associated antibiotics such as nalidixic acid, trimethoprim sulfamethoxazole, streptomycin, gentamicin, chloramphenicol and tetracycline changed progressively from the second day of treatment to the fifth day. In the control piglet group, the number of $E$. coli resistant to antibiotics remained stable. These results are in agreement with those of Sorum and Sunde (2001) and Bibbal et al. (2009), where they demonstrated the emergence of resistances of the various antibiotics associated with animal resistant digestive $E$. coli during treatment with antibiotics. This emergence of resistance to these antibiotics during periods of antibiotic treatment in animals could be explained by the presence of preexisting resistant organisms before treatment or of resistant gene transfer (Philipps et al., 2004; Sanders et al., 2011). Mentula et al. (2006) showed that when ampicillin was administered to dogs, the emergence of resistance was linked to the multiplication of $E$. coli resistant strains.

induced by administration by the intramuscular route. The different ways of administering antibiotics in animals also contribute to the selection of multidrug resistant strains. The transfer of resistant genes from resistant bacteria of animal origin to those of the commensal flora of the human digestive tract via the food chain therefore constitutes a danger to public health. 


\section{AKNOWLEDGEMENTS}

This study is a scientifical collaboration of the Pasteur Institute of Côte d'Ivoire and Nangui Abrogoua University. We thank Mrs. Nathalie Guessennd (Head of National Reference Center for Antibiotics, Pasteur Institute of Côte

\section{REFERENCES}

Belloc C, Lam DC, Pellerin JL, Beaudeau F, Laval A. 2005. Effect of quinolone treatment on selection and persistence of quinolone-resistant Escherichia coli in swine faecal flora. Journal of Applied Microbiology 99: 954-959.

Bibbal DV, Dupouy JP, Ferre PL, Toutain O, Fayet MF, Bousquet-Melou A. 2007. Impact of threeampicillin dosage regimen on selection of ampicillin resistance in Enterobacteriaceae and excretion of bla TEM genes in swine faeces. Applied and Environmental Microbiology 73: 4785-4790.

Bibbal D, Dupouy V, Prere MF, Toutain PL, BousquetMelou A. 2009. Relatedness of Escherichia coli strains with different susceptibility phenotypes isolated from swine faeces during ampicillin treatment. Applied and Environmental Microbiology 75(10):2999-3006.

CASFM. 2014. Comité de l'Antibiogramme de la Société Française de Microbiologie_ EUCAST (European Committee on Antimicrobial Susceptibility Testing). Recommandations 2014. www.sfm-microbiologie.org/UserFiles/.../ casfm/CASFM_EUCAST_V1_0_2014 (1)

FEDESA. 2000. The microbial threat. Press release on the European Union conference. FEDESA. Copenhagen, Denmark.

Jansen G, Weissing F, de Vries-Hospers H, Tonk R, Van der Waaij D. 1992. The non-enzymatic inactivation of thirteen beta-lactam antibiotics in human faeces Infection 20: 355-359.

Kempf I, Jouy E, Granier S, Bruneau M. 2014. L'antibiorésistance des bactéries isolées chez le porc (première partie). Le nouveau praticien vétérinaire, élevages et santé 6: 189-194.

Li XZ, Mehrotra M, Ghimire S, Adewoye L. 2007. BetaLactam resistance and beta-lactamases in bacteria of animal origin. Veterinary Microbiology 121: $197-214$

Livermore D. M. 1995. Beta-Lactamases in laboratory and clinical resistance. Clinical Microbiology Reviews 8: 557-584.

Mentula S, Virtanen T, Kanervo-Nordstrom A, Harmoinen $J$, Westermarck E, Rautio M, Huovinen P,
d'Ivoire), Mr. Eugène Koffi (Chief of Unity of Animal Resources Management, Pasteur Institute of Côte d'Ivoire) and Mr. Adjehi Dadié (Scientific researcher of Nangui Abrogoua University).

Kononen E. 2006. Relatedness of Escherichia coli strains with different susceptibility patterns isolated from beagle dogs during ampicillin treatment. International Journal of Antimicrobial Agents 27: 46-50.

Phillips I, Casewell M. 2004. "Does the use of antibiotics in food animals pose a risk to human health? A critical review of published data." Journal of Antimicrobial Chemother 53 (1): 28-52.

Pijpers A, Van KB, Schoevers EJ, Verheijden HM, Van Miert AM. 1989. In vitro activity of five tetracyclines and some other antimicrobial agents against four porcine respiratory tract pathogens. Journal of Veterinary and Pharmacology Therapeutic 12: 267-276.

Salyers A, Gupta A, Wang Y. 2004. Human intestinal bacteria as reservoirs for antibiotic resistance genes. Trends Microbiology 12: 412-416

Sanders P, Bousquet-Mélou A, Chauvin C, Toutain PL. 2011. Utilisation des antibiotiques en élevage et enjeux de santé publique. INRA Production Animal 24 (2): 199-204.

Sorum H and Sunde M. 2001. Resistance to antibiotics in the normal flora of animals. Veterinary Research 32: 227-241.

Sunde M, Fossum K, Solberg A, Sorum H. 1998. Antibiotic resistance in Escherichia coli of the normal intestinal flora of swine. Microbial Drug Resistance 4: 289-299.

Zhang L, Huang H. 2013. "Antibiotic administration routes significantly influence the levels of antibiotic resistance in gut microbiota. "Antimicrobial Agents Chemother 57 (8): 3659-3666. 